The Journal of $\mathbf{N}_{\text {onlinear }} \mathbf{S}_{\text {ciences and }}$ Applications http://www.tjnsa.com

\title{
REDUCTION OF AN OPERATOR EQUATION IN TO AN EQUIVALENT BIFURCATION EQUATION THROUGH SCHAUDER'S FIXED POINT THEOREM
}

\author{
PALLAV KUMAR BARUAH ${ }^{1 *}$, B V K BHARADWAJ ${ }^{2}$ AND M VENKATESULU ${ }^{3}$ \\ This paper is dedicated to Bhagawan Sri Sathya Sai Baba.
}

\begin{abstract}
In this paper we deal with the Nonlinear Coupled Ordinary Differential Equations(Nonlinear CODE). A Multipoint Boundary Value Problem(MBVP) associated with these Nonlinear Equations is defined as an Operator Equation. This equation(infinite dimensional) is reduced to an Equivalent Bifurcation Equation(finite dimensional) using Schauder's Fixed Point Theorem. This Bifurcation Equation being on a finite dimensional space can be easily solved by using standard approximation techniques.
\end{abstract}

\section{INTRODUCTION AND PRELIMINARIES}

A lot of work has been done on the Differential Operator and the associated Boundary Value Problems. Coupled Systems are very common in nature. Many real life situations can be modeled using these coupled differential equations. Some applications of such equations can be found in the prey-predator models [14] where the interdependence of the dependent variables is clearly seen. So there is a need for a unified theory for these Coupled Ordinary Differential Equations. There are various methods to analyse differential equations and show the existence of their solutoins, one such is through the fixed point theorems. Some papers in literature dealing with operators and fixed point theorems are [1, 2, 13]. We study these equations through a Functional Analytic approach. This approach was first given by Kantorovich [8]. Cesari and Locker [9, 10, 3, 4] have adopted

Date: Received: 26, Feb., 2010.

* Corresponding author

(c) 2010 N.A.G.

2000 Mathematics Subject Classification. 34G20, 34L30, 34B05.

Key words and phrases. Coupled Differential Operator, Nonlinear Operator, Hilbert Space. 
this approach for studying the nonlinear Differential Equations and the associated BVPs. It is very useful to study the linear equations first before embarking on the nonlinear equations. So we have studied the Linear Coupled Ordinary Differential Equations and the associated BVPs in [11] and [12. P C Das and Venkatesulu [5],[6], [7] have studied a MBVP associated with Nonlinear Ordinary Differential Equations using the alternative method given by Cesari [3]. We adopted the same approach to study the Nonlinear Coupled Ordinary Differential Equations and the associated MBVP.

First we have given few mathematical preliminaries useful in the study. We define the Projections $P_{m}, Q_{m}$ and the right inverse $H$. Then we prove some relations involving $H, L, P_{m}$ and $Q_{m}$. We gave an integral representation for $H$ and $H\left(I-P_{m}\right)$. In the succeeding sections we define the MBVP associated with Nonlinear CODE as an operator equation. Then we reduce this operator equation (on an infinite dimensional space) in to an equivalent Bifurcation Equation (on a finite dimensional space) using the Schauder's Fixed Point Theorem as in [6].

1.1. Mathematical Preliminaries. In this section we have given some preliminary definitions about the setting in which we are dealing the problem. We have also defined the formal coupled differential operator, maximal and the minimal operator and stated some proven results, which characterize these operators.

Definition 1.1. We denote by $L^{2}[a, b] \times L^{2}[a, b]$ a real or complex Hilbert space of ordered pairs(formed by the cartesian product) of all square integrable functions with inner product defined as

$$
\left(\left\{u_{1}, u_{2}\right\},\left\{v_{1}, v_{2}\right\}\right)=\left(u_{1}, v_{1}\right)_{1}+\left(u_{2}, v_{2}\right)_{1}=\int_{a}^{b} u_{1}(t) \overline{v_{1}(t)} d t+\int_{a}^{b} u_{2}(t) \overline{v_{2}(t)} d t
$$

and norm

$$
\left\|\left\{u_{1}, u_{2}\right\}\right\|=\left(\left\|u_{1}(t)\right\|_{1}^{2}+\left\|u_{2}(t)\right\|_{1}^{2}\right)^{1 / 2}
$$

and convergence is denoted by $\left\{u_{1}, u_{1}\right\}_{k} \rightarrow\left\{u_{1}, u_{1}\right\}$ where $(., .)_{1}$ and $\|$. $\|_{1}$ denote the inner product and norm in $L^{2}[a, b]$ defined as

$$
\left(u_{1}, u_{2}\right)=\int_{a}^{b} u_{1}(t) \overline{u_{2}(t)} d t
$$

Definition 1.2. we denote by $H^{n}[a, b]$, the subspace of $L^{2}[a, b]$, consisting of all functions $u \in C^{n-1}[a, b]$, with $u^{(n-1)}$ absolutely continuous on [a,b] and with $u^{(n)} \in L^{2}[a, b]$. Also we let

$H^{0}[a, b]=L^{2}[a, b]$.

We denote by $H^{n}[a, b] \times H^{m}[a, b]$ the cartesian product of $H^{n}[a, b]$ and $H^{m}[a, b]$, taken in that order.(i.e., It consists of all ordered pairs of the form $\{u, v\}$, where, $u \in H^{n}[a, b]$ and $\left.v \in H^{m}[a, b]\right)$ The inner product and norm are induced from the space $L^{2}[a, b] \times L^{2}[a, b]$

Definition 1.3. A formal Coupled Ordinary Differential Operator(CODO) of order $(n, \widehat{m}, \widehat{n}, m)$ on the interval $[a, b]$ is an operator of the form:

$$
\left(L_{1} M_{1} ; L_{2} M_{2}\right)(\{f, g\})=\left\{h_{1}, h_{2}\right\},
$$


such that

$$
\begin{aligned}
& L_{1}(f)+M_{1}(g)=h_{1} \\
& L_{2}(f)+M_{2}(g)=h_{2}
\end{aligned}
$$

where $\{f, g\} \in H^{n}[a, b] \times H^{m}[a, b],\left\{h_{1}, h_{2}\right\} \in L^{2}[a, b] \times L^{2}[a, b]$

$L_{1}, M_{1}, L_{2}, M_{2}$ are formal differential operators of orders $n, \widehat{m}, \widehat{n}, m$ respectively on the interval $[a, b]$, and are of the form

where all coefficients

$$
\begin{aligned}
& L_{1}=\sum_{i=0}^{n} p_{i}(t)\left(\frac{\mathrm{d}}{\mathrm{dt}}\right)^{i}, M_{1}=\sum_{i=0}^{\hat{\mathrm{m}}} \hat{\mathrm{q}}_{i}(t)\left(\frac{\mathrm{d}}{\mathrm{dt}}\right)^{i} \\
& L_{2}=\sum_{i=0}^{\hat{\mathrm{n}}} \hat{\mathrm{p}}_{i}(t)\left(\frac{\mathrm{d}}{\mathrm{dt}}\right)^{i}, M_{2}=\sum_{i=0}^{m} q_{i}(t)\left(\frac{\mathrm{d}}{\mathrm{dt}}\right)^{i}
\end{aligned}
$$

$p_{i}(i=0,1,2, \ldots \ldots, n), \widehat{q}_{i}(i=0,1,2, \ldots \ldots, \widehat{m}), \widehat{p}_{i}(i=0,1,2, \ldots \ldots, \widehat{n})$

and $q_{i}(i=0,1,2, \ldots \ldots, m)$ belong to $L^{2}[a, b]$ and $\mathrm{t} \in[a, b]$

Definition 1.4. If $\mathrm{L}$ be a $(n, n, n, n)$ ordered coupled ordinary differential operator it is said to be invertible iff $\mathrm{L}$ is one to one and on to $L^{2}[a, b] \times L^{2}[a, b]$.

The operator $\left(L_{1} M_{1}, L_{2} M_{2}\right)$ is linear and closed.

The proof of the statement can be concluded from the actual definitions of the linearity and the closedness of the operator and some of the other properties of it.

\section{Assumption 1:}

$n \geq \widehat{n}, m \geq \widehat{m}, n \geq \widehat{m}, m \geq \widehat{n}$.

Theorem 1.5. Basic Existence-Uniqueness Theorem for IVP :

Let assumption 1 be true. Also, assume that there exist positive constants $\alpha$ and $\beta$ such that $\left|p_{n}(t)\right| \geq \alpha$ and $\left|q_{m}(t)\right| \geq \beta$ for all $t \in[a, b]$. Let $h_{1}, h_{2}$ belong to $L^{2}[a, b]$, let $t_{o} \in[a, b]$ and let $c_{0}, c_{1} \ldots \ldots . ., c_{n-1}, c_{n}, \ldots \ldots, c_{n+m-1}$ be any arbitrary set of $(n+m)$ scalars. Then there exists a unique pair of functions $\{(f, g)\} \in$ $H^{n}[a, b] \times H^{m}[a, b]$ such that

$$
L_{1}(f)+M_{1}(g)=h_{1}, L_{2}(f)+M_{2}(g)=h_{2}
$$

a.e., on $[a, b]$ and satisfying the initial conditions

$$
f^{(i)}\left(t_{0}\right)=c_{i}, i=0, \ldots ., n-1
$$

and

$$
g^{(i)}\left(t_{0}\right)=c_{n+i}, i=0, \ldots ., m-1
$$

For the next results, we assume the following:

\section{Assumption 2:}

$\left(L_{1} M_{1} ; L_{2} M_{2}\right)$ is a formal coupled ordinary differential equation of order $(n, \widehat{m}, \widehat{n}, m)$ on $[a, b]$, such that the following conditions are satisfied.

Suppose that the coefficients, $p_{i},(i=0,1,2, \ldots, n), \widehat{q}_{i},(i=0,1,2, \ldots, \widehat{m}), \widehat{p}_{i}$, $(i=0,1,2, \ldots, \widehat{n}), q_{i},(i=0,1,2, \ldots, m)$ satisfy the following conditions:

$$
\begin{gathered}
p_{i} \in H^{i}[a, b], i=0,1, \ldots, n, \hat{q}_{i} \in H^{i}[a, b], i=0,1, \ldots, \widehat{m} \\
\hat{p}_{i} \in H^{i}[a, b], i=0,1, \ldots, \widehat{n}, q_{i} \in H^{i}[a, b], i=0,1, \ldots, m
\end{gathered}
$$


Now we have a definition.

Definition 1.6. Let $\left(L_{1} M_{1} ; L_{2} M_{2}\right)$ be a formal coupled ordinary differential operator of order $(n, \widehat{m}, \widehat{n}, m)$ on $[a, b]$, such that assumptions 1 and 2 are satisfied.The formal coupled ordinary differential operator

$$
\left(L_{1} M_{1} ; L_{2} M_{2}\right)^{*}=\left(L_{1}^{*} L_{2}^{*} ; M_{1}^{*} M_{2}^{*}\right)
$$

is called the formal adjoint of $\left(L_{1} M_{1} ; L_{2} M_{2}\right)$ where

$$
\begin{aligned}
& L_{1}^{*}=\sum_{j=0}^{n} p_{j}^{*}(t)\left(\frac{\mathrm{d}}{\mathrm{dt}}\right)^{j} \text { where } p_{j}^{*} \in H^{j}[a, b] \text { for } j=0,1,2, \ldots . n . \\
& \text { with } p_{j}^{*}(t)=\sum_{i=j}^{n}(-1)^{i}\left(\begin{array}{l}
i \\
j
\end{array}\right)\left(\frac{\mathrm{d}}{\mathrm{dt}}\right)^{i-j} \overline{p_{i}(t)}, j=0,1,2, \ldots ., n-1 \text {. } \\
& M_{1}^{*}=\sum_{j=0}^{\hat{\mathrm{m}}} \hat{\mathrm{q}}_{j}^{*}(t)\left(\frac{\mathrm{d}}{\mathrm{dt}}\right)^{j} \text { where } \hat{\mathrm{q}}_{j}^{*} \in H^{j}[a, b] \text { for } j=0,1,2, \ldots . \hat{\mathrm{m}} \\
& \text { with } \hat{\mathrm{q}}_{j}^{*}(t)=\sum_{i=j}^{\hat{\mathrm{m}}}(-1)^{i}\left(\begin{array}{l}
i \\
j
\end{array}\right)\left(\frac{\mathrm{d}}{\mathrm{dt}}\right)^{i-j} \overline{\left(\hat{\mathrm{q}}_{i}(t)\right)}, j=0,1,2, \ldots ., \hat{\mathrm{m}}-1 \text {. } \\
& L_{2}^{*}=\sum_{j=0}^{\hat{\mathrm{n}}} \hat{\mathrm{p}}_{j}^{*}(t)\left(\frac{\mathrm{d}}{\mathrm{dt}}\right)^{j} \text { where } \hat{\mathrm{p}}_{j}^{*} \in H^{j}[a, b] \text { for } j=0,1,2, \ldots . \hat{\mathrm{n}} \\
& \text { with } \hat{\mathrm{p}}_{j}^{*}(t)=\sum_{i=j}^{\hat{\mathrm{n}}}(-1)^{i}\left(\begin{array}{l}
i \\
j
\end{array}\right)\left(\frac{\mathrm{d}}{\mathrm{dt}}\right)^{i-j} \overline{\left(\hat{\mathrm{p}}_{i}(t)\right)}, j=0,1,2, \ldots ., \hat{\mathrm{n}}-1 \text {. } \\
& M_{2}^{*}=\sum_{j=0}^{n} q_{j}^{*}(t)\left(\frac{\mathrm{d}}{\mathrm{dt}}\right)^{j} \text { where } q_{j}^{*} \in H^{j}[a, b] \text { for } j=0,1,2, \ldots . m . \\
& \text { with } q_{j}^{*}(t)=\sum_{i=j}^{m}(-1)^{i}\left(\begin{array}{l}
i \\
j
\end{array}\right)\left(\frac{\mathrm{d}}{\mathrm{dt}}\right)^{i-j} \overline{\left(q_{i}(t)\right)}, j=0,1,2, \ldots . ., m-1 \text {. }
\end{aligned}
$$

In case $\left(L_{1} M_{1} ; L_{2} M_{2}\right)=\left(L_{1} M_{1} ; L_{2} M_{2}\right)^{*}$, we say that $\left(L_{1} M_{1} ; L_{2} M_{2}\right)$ is formally self-adjoint.

Now we define few norms which are used throughout this paper.

\section{Definition 1.7.}

$$
\begin{gathered}
\|\{x, \hat{x}\}\|_{H^{n}[a, b] \times H^{n}[a, b]}=\sqrt{b-a} \sum_{i=0}^{n-1} \sup _{t \in[a, b]}\left|\left\{x^{i}, \hat{x}^{i}\right\}\right|+\left\|\left\{x^{n}, \hat{x}^{n}\right\}\right\| \\
\mu(\{x, \hat{x}\})=\max \left(\max _{i=0 . . n-2} \sup _{t \in[a, b]}\left|\left\{x^{i}, \hat{x}^{i}\right\}\right|, \text { ess.sup }_{t \in[a, b]}\left|\left\{x^{n}, \hat{x}^{n}\right\}\right|\right)
\end{gathered}
$$

Let $\tau$ be the formal coupled differential operator defined above.

For each $j=1,2, \ldots, k$ we define,

$$
B_{j}\left\{x_{1}, x_{2}\right\}=\sum_{l=1}^{2} \sum_{i=0}^{n-1}\left(\alpha_{0 j i}^{l} x_{l}^{i}(a)+\alpha_{1 j i}^{l} x_{l}^{i}\left(a_{1}\right)+\ldots+\alpha_{h j i}^{l} x_{l}^{i}(b)\right)
$$

where the interval $[a, b]$ is divided as $a=a_{0} \leq a_{1} \leq \ldots \leq a_{h}=b$. We assume that $k \leq n$ and all $B_{j}$ s are linearly independent.

So let us define the Linear Coupled Ordinary Differential Operator $L$ here as the operator generated by the formal coupled ordinary differential operator and the boundary conditions in the equation (1.4).

Then let the linear operators $T_{1}(\tau)$ and $T_{0}(\tau)$ be defined as follows

$$
\begin{gathered}
D\left(T_{1}(\tau)\right)=\left\{\{x, y\} \in H^{n}[a, b] \times H^{n}[a, b]\right\} \\
T_{1}(\tau)\{x, y\}=\tau\{x, y\}
\end{gathered}
$$


and

$$
\begin{gathered}
D\left(T_{0}(\tau)\right)=\left\{\{x, y\} \in H_{0}^{n}[a, b] \times H_{0}^{n}[a, b]\right\} \\
T_{0}(\tau)\{x, y\}=\tau\{x, y\}
\end{gathered}
$$

Clearly, $T_{1}(\tau)$ is an extension of both $T_{0}(\tau)$ and $L$.

Moreover, $T_{1}(\tau)$ is the adjoint of the operator $T_{o}\left(\tau^{*}\right)$ where $\tau^{*}$ represents the formal adjoint of the operator $\tau$.

Now let us recollect the following well known facts(Proved in [11]) about $L$ :

$D(L)$ is dense in $L^{2}[a, b] \times L^{2}[a, b]$.

$L$ is a closed linear operator.

$R(L)$ is closed in $L^{2}[a, b] \times L^{2}[a, b]$.

$L^{2}[a, b] \times L^{2}[a, b]=R(L) \oplus N\left(L^{*}\right)$ where $N\left(L^{*}\right)$ denotes the null space of the adjoint of $L$.

We know that $N\left(T_{1}(\tau)\right)$ is $n$-dimensional with $N(L) \subseteq N\left(T_{1}(\tau)\right)$. Let us choose function pairs $\left\{\phi_{i}, \widehat{\phi}_{i}\right\}, i=1,2, \ldots, n$. in $C^{\infty}[a, b]$ to form an orthonormal basis for $N\left(T_{1}(\tau)\right)$ in such a way that $\left\{\phi_{i}, \widehat{\phi}_{i}\right\}, i=1,2, \ldots, p$. forms an orthonormal basis for $N(L)$. we also chose $\left\{\omega_{i}, \widehat{\omega}_{i}\right\}, i=1,2, . ., q$ in $D\left(L^{*}\right)$ to form an orthonormal basis for $N\left(L^{*}\right)$.

We note that the operator $L / D(L) \cap N(L)^{\perp}$ is a one-to-one closed linear operator having the same range as $L$. Now Let $H$ denote the inverse of this operator.

$$
H=\left\{L / D(L) \cap N(L)^{\perp}\right\}^{-1}
$$

By the closed graph theorem, $H$ is a one-to-one continuous linear operator. Clearly, $D(H)=R(L), R(H)=L / D(L) \cap N(L)^{\perp}$.

Moreover,

$$
L H\left\{y_{1}, y_{2}\right\}=\left\{y_{1}, y_{2}\right\}
$$

for all $\left\{y_{1}, y_{2}\right\} \in R(L)$

and

$$
H L\left\{x_{1}, x_{2}\right\}=\left\{x_{1}, x_{2}\right\}-\sum_{i=1}^{p}\left(\left\{x_{1}, x_{2}\right\},\left\{\phi_{i}, \widehat{\phi}_{i}\right\}\right)\left\{\phi_{i}, \widehat{\phi}_{i}\right\},
$$

for all $\left\{x_{1}, x_{2}\right\} \in D(L)$

Thus $H$ is a continuous right inverse of $L$.

1.2. Projections $P_{m}$ and $Q_{m}$ and their Relation with $L$ and $H$. We assume that there exist elements $\left\{\omega_{q+1}, \widehat{\omega_{q+1}}\right\},\left\{\omega_{q+2}, \widehat{\omega_{q+2}}\right\}, \ldots,\left\{\omega_{m}, \widehat{\omega_{m}}\right\} \ldots$ belonging to $D\left(L^{*}\right)$ such that the sequence of functions $\left\{\omega_{1}, \widehat{\omega_{1}}\right\},\left\{\omega_{2}, \widehat{\omega_{2}}\right\}, \ldots$, $\left\{\omega_{q}, \widehat{\omega_{q}}\right\}\left\{\omega_{q+1}, \widehat{\omega_{q+1}}\right\},\left\{\omega_{q+2}, \widehat{\omega_{q+2}}\right\}, \ldots .,\left\{\omega_{m}, \widehat{\omega_{m}}\right\} \ldots$ form a complete orthonormal set in $L^{2}[a, b] \times L^{2}[a, b]$. Since $L^{2}[a, b] \times L^{2}[a, b]=R(L) \oplus N\left(L^{*}\right)$, the elements $\left\{\omega_{q+1}, \widehat{\omega_{q+1}}\right\},\left\{\omega_{q+2}, \widehat{\omega_{q+2}}\right\}, \ldots .,\left\{\omega_{m}, \widehat{\omega_{m}}\right\} \ldots$ belong to $R(L)$. Hence $H\left\{\omega_{q+i}, \widehat{\omega_{q+i}}\right\}$, $i \geq 1$ are defined and belong to $D(L) \cap N(L)^{\perp}$. Let $S_{o}$ be the subspace spanned by the elements $\left\{\phi_{i}, \widehat{\phi}_{i}\right\}, i=1,2, \ldots, p$. and $H\left\{\omega_{q+i}, \widehat{\omega_{q+i}}\right\}, i=1,2, \ldots, m$. 
Now we define the sequences of projections $P_{m}$ and $Q_{m}$ as

where $m>q$.

$$
\begin{aligned}
& P_{m}\{x, \widehat{x}\}=\sum_{i=1}^{m}\left(\{x, \widehat{x}\},\left\{\omega_{i}, \widehat{\omega}_{i}\right\}\right)\left\{\omega_{i}, \widehat{\omega}_{i}\right\} \\
& Q_{m}\{x, \widehat{x}\}=\sum_{i=1}^{p}\left(\{x, \widehat{x}\},\left\{\phi_{i}, \widehat{\phi}_{i}\right\}\right)\left\{\phi_{i}, \widehat{\phi}_{i}\right\} \\
& \quad+\sum_{i=q+1}^{m}\left(\{x, \widehat{x}\}, L^{*}\left\{\omega_{i}, \widehat{\omega}_{i}\right\}\right) H\left\{\omega_{i}, \widehat{\omega}_{i}\right\}
\end{aligned}
$$

These operators $P_{m}, Q_{m}$ have the following properties.

$P_{m}$ and $Q_{m}$ are continuous linear operators on all of $L^{2}[a, b] \times L^{2}[a, b]$.

$R\left(P_{m}\right)=<\omega_{1}, \omega_{2}, \ldots . ., \omega_{m}>$.

$R\left(Q_{m}\right)=S_{0} \subset D(L)$.

$P_{m}^{2}=P_{m}$ and $Q_{m}^{2}=Q_{m}$.

The range of $\left(I-P_{m}\right)$ is a subset of $R(L)$ and $H\left(I-P_{m}\right)$ is a continuous linear operator defined on all of $S$.

Proofs of these statements are very trivial from the definitions.

We now prove a theorem which we use in the subsequent discussions.

Theorem 1.8. The following results are valid:

(1) $H\left(I-P_{m}\right) L\left\{x_{1}, x_{2}\right\}=\left(I-Q_{m}\right)\left\{x_{1}, x_{2}\right\}$ for all $\left\{x_{1}, x_{2}\right\} \in D(L)$.

(2) $L H\left(I-P_{m}\right)\left\{x_{1}, x_{2}\right\}=\left(I-P_{m}\right)\left\{x_{1}, x_{2}\right\}$ for all $\left\{x_{1}, x_{2}\right\} \in S$.

(3) $L Q_{m}\left\{x_{1}, x_{2}\right\}=P_{m} L\left\{x_{1}, x_{2}\right\}$ for all $\left\{x_{1}, x_{2}\right\} \in D(L)$.

(4) $Q_{m} H\left(I-P_{m}\right)\left\{x_{1}, x_{2}\right\}=0$ for all $\left\{x_{1}, x_{2}\right\} \in S$.

Proof. (1) Let $\left\{x_{1}, x_{2}\right\} \in D(L)$. Then

$$
\begin{gathered}
\left(I-P_{m}\right)\left\{x_{1}, x_{2}\right\}=L\left\{x_{1}, x_{2}\right\}-\sum_{i=1}^{m}\left(L\left\{x_{1}, x_{2}\right\},\left\{\omega_{i}, \hat{\omega}_{i}\right\}\right)\left\{\omega_{i}, \hat{\omega}_{i}\right\} \\
=L\left\{x_{1}, x_{2}\right\}-\sum_{i=1}^{m}\left(\left\{x_{1}, x_{2}\right\}, L^{*}\left\{\omega_{i}, \hat{\omega}_{i}\right\}\right)\left\{\omega_{i}, \hat{\omega}_{i}\right\} \\
=L\left\{x_{1}, x_{2}\right\}-\sum_{i=q+1}^{m}\left(\left\{x_{1}, x_{2}\right\}, L^{*}\left\{\omega_{i}, \hat{\omega}_{i}\right\}\right)\left\{\omega_{i}, \hat{\omega}_{i}\right\}
\end{gathered}
$$

Therefore

$$
\begin{aligned}
H\left(I-P_{m}\right) L\left\{x_{1}, x_{2}\right\}= & H L\left\{x_{1}, x_{2}\right\} \\
& -\sum_{i=q+1}^{m}\left(\left\{x_{1}, x_{2}\right\}, L^{*}\left\{\omega_{i}, \hat{\omega}_{i}\right\}\right) H\left\{\omega_{i}, \hat{\omega}_{i}\right\} \\
= & \left\{x_{1}, x_{2}\right\}-\sum_{i=1}^{p}\left(\left\{x_{1}, x_{2}\right\},\left\{\phi_{i}, \hat{\phi}_{i}\right\}\right)\left\{\phi_{i}, \hat{\phi}_{i}\right\} \\
& -\sum_{i=q+1}^{m}\left(\left\{x_{1}, x_{2}\right\}, L^{*}\left\{\omega_{i}, \hat{\omega}_{i}\right\}\right)\left\{\omega_{i}, \hat{\omega}_{i}\right\} \\
= & \left(I-Q_{m}\right)\left\{x_{1}, x_{2}\right\} .
\end{aligned}
$$


(2) Since $\left(I-P_{m}\right)\left\{x_{1}, x_{2}\right\} \in R(L)$ for all $\left\{x_{1}, x_{2}\right\} \in L^{2}[a, b] \times L^{2}[a, b]$, we have

$L H\left(I-P_{m}\right)\left\{x_{1}, x_{2}\right\}=\left(I-P_{m}\right)\left\{x_{1}, x_{2}\right\}$

(3) Let $\left\{x_{1}, x_{2}\right\} \in D(L)$. Then

$$
\begin{aligned}
Q_{m}\left\{x_{1}, x_{2}\right\}= & \sum_{i=1}^{p}\left(\left\{x_{1}, x_{2}\right\},\left\{\phi_{i}, \hat{\phi}_{i}\right\}\right)\left\{\phi_{i}, \hat{\phi}_{i}\right\}+ \\
& \sum_{i=q+1}^{m}\left(\left\{x_{1}, x_{2}\right\}, L^{*}\left\{\omega_{i}, \hat{\omega}_{i}\right\}\right) H\left\{\omega_{i}, \hat{\omega}_{i}\right\}
\end{aligned}
$$

Therefore

$$
\begin{aligned}
L Q_{m}\left\{x_{1}, x_{2}\right\}= & \sum_{i=1}^{p}\left(\left\{x_{1}, x_{2}\right\},\left\{\phi_{i}, \hat{\phi}_{i}\right\}\right) L\left\{\phi_{i}, \hat{\phi}_{i}\right\}+ \\
& \sum_{i=q+1}^{m}\left(\left\{x_{1}, x_{2}\right\}, L^{*}\left\{\omega_{i}, \hat{\omega}_{i}\right\}\right) L H\left\{\omega_{i}, \hat{\omega}_{i}\right\} \\
= & \sum_{i=q+1}^{m}\left(\left\{x_{1}, x_{2}\right\}, L^{*}\left\{\omega_{i}, \hat{\omega}_{i}\right\}\right) L H\left\{\omega_{i}, \hat{\omega}_{i}\right\}
\end{aligned}
$$

Thus

$$
\begin{aligned}
L Q_{m}\left\{x_{1}, x_{2}\right\}=\sum_{i=1}^{m} & \left(\left\{x_{1}, x_{2}\right\}, L^{*}\left\{\omega_{i}, \hat{\omega}_{i}\right\}\right) L H\left\{\omega_{i}, \hat{\omega}_{i}\right\} \\
= & \sum_{i=1}^{m}\left(L\left\{x_{1}, x_{2}\right\},\left\{\omega_{i}, \hat{\omega}_{i}\right\}\right)\left\{\omega_{i}, \hat{\omega}_{i}\right\} \\
& =P_{m} L\left\{x_{1}, x_{2}\right\}
\end{aligned}
$$

(4) Let $\left\{x_{1}, x_{2}\right\} \in L^{2}[a, b] \times L^{2}[a, b]$. Then

$$
\begin{aligned}
H\left(I-P_{m}\right)\left\{x_{1}, x_{2}\right\} & =H\left(\left\{x_{1}, x_{2}\right\}-\sum_{j=1}^{m}\left(\left\{x_{1}, x_{2}\right\},\left\{\omega_{j}, \hat{\omega}_{j}\right\}\right)\left\{\omega_{j}, \hat{\omega}_{j}\right\}\right) \\
& =H\left\{x_{1}, x_{2}\right\}-\sum_{j=1}^{m}\left(\left\{x_{1}, x_{2}\right\},\left\{\omega_{j}, \hat{\omega}_{j}\right\}\right) H\left\{\omega_{j}, \hat{\omega}_{j}\right\}
\end{aligned}
$$

Therefore

$$
\begin{aligned}
& Q_{m} H\left(I-P_{m}\right)\left\{x_{1}, x_{2}\right\} \\
& =Q_{m} H\left\{x_{1}, x_{2}\right\}-Q_{m}\left(\sum_{j=1}^{m}\left(\left\{x_{1}, x_{2}\right\},\left\{\omega_{j}, \hat{\omega}_{j}\right\}\right) H\left\{\omega_{j}, \hat{\omega}_{j}\right\}\right) \\
& =\sum_{i=1}^{p}\left(H\left\{x_{1}, x_{2}\right\},\left\{\phi_{i}, \hat{\phi}_{i}\right\}\right)\left\{\phi_{i}, \hat{\phi}_{i}\right\}+\sum_{i=q+1}^{m}\left(H\left\{x_{1}, x_{2}\right\}, L^{*}\left\{\omega_{i}, \hat{\omega}_{i}\right\}\right) H\left\{\omega_{i}, \hat{\omega}_{i}\right\} \\
& -\sum_{i=1}^{p}\left(\sum_{j=1}^{m}\left(\left\{x_{1}, x_{2}\right\},\left\{\omega_{j}, \hat{\omega}_{j}\right\}\right) H\left\{\omega_{j}, \hat{\omega}_{j}\right\},\left\{\phi_{i}, \hat{\phi}_{i}\right\}\right)\left\{\phi_{i}, \hat{\phi}_{i}\right\} \\
& -\sum_{i=q+1}^{m}\left(\sum_{j=1}^{m}\left(\left\{x_{1}, x_{2}\right\},\left\{\omega_{j}, \hat{\omega}_{j}\right\}\right) H\left\{\omega_{j}, \hat{\omega}_{j}\right\}, L^{*}\left\{\omega_{i}, \hat{\omega}_{i}\right\}\right) H\left\{\omega_{i}, \hat{\omega}_{i}\right\} \\
& =\sum_{i=q+1}^{m}\left(H\left\{x_{1}, x_{2}\right\}, L^{*}\left\{\omega_{i}, \hat{\omega}_{i}\right\}\right) H\left\{\omega_{i}, \hat{\omega}_{i}\right\}
\end{aligned}
$$




$$
\begin{aligned}
& -\sum_{i=q+1}^{m}\left(\sum_{j=1}^{m}\left(\left\{x_{1}, x_{2}\right\},\left\{\omega_{j}, \hat{\omega}_{j}\right\}\right)\left\{\omega_{j}, \hat{\omega}_{j}\right\},\left\{\omega_{i}, \hat{\omega}_{i}\right\}\right) H\left\{\omega_{i}, \hat{\omega}_{i}\right\} \\
& \text { Thus } \\
& Q_{m} H\left(I-P_{m}\right)\left\{x_{1}, x_{2}\right\}=0(\text { i.e }\{0,0\})
\end{aligned}
$$

Now we give an integral representation to $H$ and $H\left(I-P_{m}\right)$.

The right inverse operator $H$, being a one-to-one continous linear operator has an integral representation given by

$$
\left(H\left\{y_{1}, y_{2}\right\}\right)(t)=\int_{a}^{b} K(t, s)\left\{y_{1}, y_{2}\right\}^{T}
$$

where $K(t, s)$ is called the Generalise Green's function.

Similarly $H\left(I-P_{m}\right)$ is also given the following representation

$$
\left(H\left(I-P_{m}\right)\left\{x_{1}, x_{2}\right\}\right)(t)=\int_{a}^{b} K_{m}(t, s)\left\{x_{1}(s), x_{2}(s)\right\}^{T} d s
$$

where $K_{m}(t, s)$ be the function defined by

$K_{m}(t, s)=$

$$
K(t, s)-\sum_{i=1}^{n}\left(\int_{a}^{b} K(t, \xi)\left\{\omega_{i}(\xi), \hat{\omega}_{i}(\xi)\right\}^{T} d \xi\right)^{T}\left\{\omega_{i}(s), \hat{\omega}_{i}(s)\right\}, a \leq t, s \leq b
$$

\section{MAin RESUlts}

2.1. The MBVP, Assumptions and Inequalities. In this section we follow the notations of the previous one. We deal with the the Nonlinear Multipoint Boundary Value Problem(MBVP) $L\{x, \hat{x}\}=N\{x, \hat{x}\}$, where $N$ is defined subsequently. Indeed we reduce the equation $L\{x, \hat{x}\}=N\{x, \hat{x}\}$ to an equivalent bifurcation equation by making use of the Schauder's fixed point theorems. We reduce the equation $L\{x, \hat{x}\}=N\{x, \hat{x}\}$ under the following assumption We assume the following for the rest of our study.

(1) $L$ satisfies all the assumptions of the previous section.

(2) Let $\left\{X\left(t, x_{0}, x_{1}, \ldots, x_{n-1}\right), \hat{X}\left(t, \hat{x}_{0}, \hat{x}_{1}, \ldots, \hat{x}_{n-1}\right)\right\}$ be a pair of nonlinear real valued function defined on the interval $[a, b]$.and $\left|\left\{x_{i}, \hat{x}_{i}\right\}\right| \leq R_{i}, i=$ $0,1, \ldots, n-1$.

(3) $\left\{X\left(t, x_{0}, x_{1}, \ldots, x_{n-1}\right), \hat{X}\left(t, \hat{x}_{0}, \hat{x}_{1}, \ldots, \hat{x}_{n-1}\right)\right\} \in L^{2}[a, b] \times L^{2}[a, b]$ for each fixed $\left\{x_{i}, \hat{x}_{i}\right\}$ satisfying $\left|\left\{x_{i}, \hat{x}_{i}\right\}\right| \leq R_{i}, i=0,1, \ldots, n-1$.

(4) There exists a real number $k_{0} \geq 0$ such that for $\left|\left\{x_{i}, \hat{x}_{i}\right\}\right| \leq R_{i}$ and $\left|\left\{y_{i}, \hat{y}_{i}\right\}\right| \leq R_{i}$, the pair of functions $\{X, \hat{X}\}$ satisfies the following 


$$
\begin{aligned}
& \|\left\{X\left(t, x_{0}, x_{1}, \ldots, x_{n-1}\right), \hat{X}\left(t, \hat{x}_{0}, \hat{x}_{1}, \ldots, \hat{x}_{n-1}\right)\right\} \\
& -\left\{X\left(t, y_{0}, y_{1}, \ldots, y_{n-1}\right), \hat{X}\left(t, \hat{y}_{0}, \hat{y}_{1}, \ldots, \hat{y}_{n-1}\right)\right\} \| \leq k_{0}\left(\sum_{i=0}^{n-1}\left|\left\{x_{i}, \hat{x}_{i}\right\}-\left\{y_{i}, \hat{y}_{i}\right\}\right|\right) \\
& \quad, t \in[a, b] .
\end{aligned}
$$

We now define the operator $N$ as following

Definition 2.1. $D(N)=\left\{\{x, \hat{x}\} \in H^{n-1}[a, b] \times H^{n-1}[a, b]: \sup _{t \in[a, b]}\left|\left\{x^{i}, \hat{x}^{i}\right\}\right| \leq\right.$ $R_{i}$

$i=0, . ., n-2$, ess.sup $\left.t \in[a, b]\left|\left\{x^{n-1}, \hat{x}^{n-1}\right\}\right| \leq R_{n-1}\right\}$

$N\{x(t), \hat{x}(t)\}=\left\{X\left(t, x(t), x^{1}(t), \ldots, x^{n-1}(t)\right), \hat{X}\left(t, \hat{x}_{0}, \hat{x}_{1}, \ldots, \hat{x}_{n-1}\right)\right\}$ for all $t \in$ $[a, b]$ for which $\left|\left\{x^{n-1}, \hat{x}^{n-1}\right\}\right| \leq R_{n-1}$

Clearly we can see that $N\{x, \hat{x}\} \in L^{2}[a, b] \times L^{2}[a, b]$ for $\{x, \hat{x}\} \in D(N)$.

Now we develop an existential theory for the Nonlinear multipoint boundary value problem(NL MBVP)

$$
L\{x, \hat{x}\}=N\{x, \hat{x}\}
$$

Now we prove some inequalities useful in our analysis.

$\|N\{x, \hat{x}\}-N\{y, \hat{y}\}\|=$

$$
\begin{aligned}
& \|\left\{X\left(., x(.), x^{1}(.), \ldots, x^{n-1}(.)\right), \hat{X}\left(., \hat{x}(.), \hat{x}^{1}(.), \ldots, \hat{x}^{n-1}(.)\right)\right\} \\
&-\left\{X\left(., y(.), y^{1}(.), \ldots, y^{n-1}(.)\right), \hat{X}\left(., \hat{y}(.), \hat{y}^{1}(.), \ldots, \hat{y}^{n-1}(.)\right)\right\} \| \\
& \leq k_{0}\left\|\left(\sum_{i=0}^{n-1}\left|\left\{x^{i}, \hat{x}^{i}\right\}-\left\{y^{i}, \hat{y}^{i}\right\}\right|\right)\right\| \\
& \leq k_{0}\left(\sum_{i=0}^{n-1}\left\|\left\{x^{i}, \hat{x}^{i}\right\}-\left\{y^{i}, \hat{y}^{i}\right\}\right\|\right) \\
& \leq k_{0}\left\{\sqrt{b-a}\left\{\sum_{i=0}^{n-2} \sup _{t \in[a, b]}\left|\left\{x^{i}, \hat{x}^{i}\right\}-\left\{y^{i}, \hat{y}^{i}\right\}\right|\right\}+\right. \\
&\left.\left\|\left\{x^{n-1}, \hat{x}^{n-1}\right\}-\left\{y^{n-1}, \hat{y}^{n-1}\right\}\right\|\right\}
\end{aligned}
$$

$\leq k_{0}\|\{x, \hat{x}\}-\{y, \hat{y}\}\|_{H^{n}[a, b] \times H^{n}[a, b]}$

where $\|\cdot\|_{H^{n}[a, b] \times H^{n}[a, b]}$ is the norm also on $H^{n-1}[a, b] \times H^{n-1}[a, b]$.

Thus for $\{x, \hat{x}\},\{y, \hat{y}\} \in D(N)$ we have

$$
\|N\{x, \hat{x}\}-N\{y, \hat{y}\}\| \leq k_{0}\|\{x, \hat{x}\}-\{y, \hat{y}\}\|_{H^{n}[a, b] \times H^{n}[a, b]}
$$

Let

$$
\rho_{m}^{i}=\left(\sup _{t \in[a, b]} \int_{a}^{b}\left|\left(\frac{\partial^{i} K_{m}(t, s)}{\partial t^{i}}\right)\right|^{2} d s\right)^{\frac{1}{2}}, i=0,1, \ldots, n-2
$$

and

$$
\rho_{m}^{n-1}=\left(\int_{a} b \int_{a}^{b}\left|\left(\frac{\partial^{n-1} K_{m}(t, s)}{\partial t^{n-1}}\right)\right|^{2} d s d t\right)^{\frac{1}{2}}
$$


Noting that $\left.\int_{a}^{b}\left|\left(\frac{\partial^{i} K_{m}(t, s)}{\partial t^{i}}\right)\right|^{2} d s\right)^{\frac{1}{2}}$ is a monotone decreasing sequence for every $t$, Dini's theorem assures uniform convergence of this sequence to zeros $m \rightarrow \infty$ for $i=0,1, \ldots, n-1$. Thus $\rho_{m}^{i} \rightarrow 0$ as $m \rightarrow \infty, i=0,1, \ldots, n-1$ and $\rho_{m}^{n-1} \rightarrow 0$ as $m \rightarrow \infty$.

So define

$$
\begin{gathered}
\theta_{m}=\sqrt{b-a}\left(\sum_{i=0}^{n-2} \rho_{m}^{i}\right)+\rho_{m}^{n-1} \\
\bar{\theta}_{m}=\max _{i=0,1, . ., n-1} \rho_{m}^{i}
\end{gathered}
$$

We observe that both $\theta_{m}, \bar{\theta}_{m}$ tend to zero as $m \rightarrow \infty$. let $X$ be the vector with the pair of functions $\{x, \hat{x}\}$. Then

$$
\begin{aligned}
& \left\|\int_{a}^{b} K_{m}(., s) X(s) d s\right\|_{H^{n}[a, b] \times H^{n}[a, b]}= \\
& \sqrt{b-a}\left(\sum_{i-0}^{n-2} \sup _{t \in[a, b]}\left|\int_{a}^{b}\left(\frac{\partial^{i} K_{m}(t, s)}{\partial t^{i}}\right) X(s) d s\right|\right)+\left\|\int_{a}^{b}\left(\frac{\partial^{n-1} K_{m}(t, s)}{\partial t^{n-1}}\right) X(s) d s\right\| \\
& \leq \sqrt{b-a}\left(\sum_{i=0}^{n-2}\left(\sup _{t \in[a, b]}\left|\int_{a}^{b}\left(\frac{\partial^{i} K_{m}(t, s)}{\partial t^{i}}\right)^{2} d s\right|\right)^{\frac{1}{2}}\|X\|\right) \\
& +\left(\int_{a}^{b} \int_{a}^{b}\left(\frac{\partial^{n-1} K_{m}(t, s)}{\partial t^{n-1}}\right)^{2} d s d t\right)^{\frac{1}{2}}\|X\|
\end{aligned}
$$

(by Schwartz inequality)

$$
\begin{aligned}
& =\left(\sqrt{b-a}\left(\sum_{i=0}^{n-2} \rho_{m}^{i}\right)+\rho_{m}^{n-1}\right)\|X\| \\
& =\theta_{m}\|X\|
\end{aligned}
$$

Hence for all $X \in L^{2}[a, b] \times L^{2}[a, b]$ we have

$$
\left\|\int_{a}^{b} K_{m}(., s) X(s) d s\right\|_{H^{n}[a, b] \times H^{n}[a, b]} \leq \theta_{m}\|X\|
$$

Also

$$
\begin{aligned}
& \mu\left(\int_{a}^{b} K_{m}(., s) X(s) d s\right)=\max _{i=0,1 . . n-1} \sup _{t \in[a, b]}\left|\int_{a}^{b} \frac{\partial^{i} K_{m}(t, s)}{\partial t^{i}} X(s) d s\right| \\
\leq & \max _{i=0,1 . . n-1}\left(\sup _{t \in[a, b]} \int_{a}^{b}\left|\frac{\partial^{i} K_{m}(t, s)}{\partial t^{i}}\right|^{2} d s\right)^{\frac{1}{2}}\|X\|
\end{aligned}
$$

(by Schwartz inequality)

$$
=\left(\max _{i=0,1, . ., n-1} \rho_{m}^{i}\right)\|X\|
$$

(by it's definition)

$=\bar{\theta}_{m}\|X\|$

Therefore for $X \in L^{2}[a, b] \times L^{2}[a, b]$ we have

$$
\mu\left(\int_{a}^{b} K_{m}(., s) X(s) d s\right) \leq \bar{\theta}_{m}\|X\|
$$

2.2. Construction of Sets $V$ and $S_{0}$. Let us consider the Banach Space $H^{n-1}[a, b] \times$ $H^{n-1}[a, b]$. We observe that the set $\widetilde{H}^{n-1}[a, b] \times \widetilde{H}^{n-1}[a, b]$ is a linear manifold of $H^{n-1}[a, b] \times H^{n-1}[a, b]$. We remember the function $\mu$ defined on $\widetilde{H}^{n-1}[a, b] \times$ $\widetilde{H}^{n-1}[a, b]$.

We consider the $p+m-q$ dimensional space $S_{0}$. Clearly $S_{0} \subset D(L) \subset H^{n}[a, b] \times$ 
$H^{n}[a, b]$. We chose $\left\{x_{0}, \hat{x}_{0}\right\} \in S_{0}$ such that $\beta=\mu\left(\left\{x_{0}, \hat{x}_{0}\right\}\right)<R$ where $R=$ $\min _{i=0, \ldots, n-1} R_{i}$. Here $R_{i}$ s are the constants defined previously in the assumptions. Let $\left\{z_{0}, \hat{z}_{0}\right\}=H\left(I-P_{m}\right) N\left\{x_{0}, \hat{x}_{0}\right\}$, and let $e$ and $\bar{e}$ be real numbers such that

$$
\left\|\left\{z_{0}, \hat{z}_{0}\right\}\right\|_{H^{n}[a, b] \times H^{n}[a, b]} \leq e, \quad \mu\left(\left\{z_{0}, \hat{z}_{0}\right\}\right) \leq \bar{e}
$$

Let $c, d, r$ and $\bar{R}$ be real numbers such that

$$
0<c<d, \quad 0<r<\bar{R}, \quad c+e<d, \bar{R}+\beta \leq R, \quad r+\bar{e}<\bar{R}
$$

The sets $V$ and $\widetilde{S}_{0}$ in $\widetilde{H}^{n-1}[a, b] \times \widetilde{H}^{n-1}[a, b]$ are defined as follows:

Definition 2.2. $V=\{\{x, \hat{x}\} \in$

$$
\left.S_{0}:\left\|\{x, \hat{x}\}-\left\{x_{0}, \hat{x}_{0}\right\}\right\|_{H^{n}[a, b] \times H^{n}[a, b]} \leq c \text { and } \mu\left(\{x, \hat{x}\}-\left\{x_{0}, \hat{x}_{0}\right\}\right) \leq r\right\}
$$

and $\widetilde{S}_{0}=\left\{\{x, \hat{x}\} \in \widetilde{H}^{n}[a, b] \times \widetilde{H}^{n}[a, b]:\right.$

$$
\left.\left\|\{x, \hat{x}\}-\left\{x_{0}, \hat{x}_{0}\right\}\right\|_{H^{n}[a, b] \times H^{n}[a, b]} \leq d \text { and } \mu\left(\{x, \hat{x}\}-\left\{x_{0}, \hat{x}_{0}\right\}\right) \leq \bar{R}\right\}
$$

We observe that $V$ is a closed and bounded subset of $L^{2}[a, b] \times L^{2}[a, b]$. Indeed, let $\left\{\left\{y_{k}, \hat{y}_{k}\right\}\right\}$ be any sequence contained in $V$ and let $\left\{\left\{y_{k}, \hat{y}_{k}\right\}\right\}$ converge to $\{y, \hat{y}\}$ in the topology of $L^{2}[a, b] \times L^{2}[a, b]$.

Firstly, we notice that $\left\{\left\{y_{k}, \hat{y}_{k}\right\}\right\} \in H^{n}[a, b] \times H^{n}[a, b]$. Since $S_{0}$ is finite dimensional, the element $\{y, \hat{y}\} \in S_{0}$. From the fact that the linear operators on a finite dimensional space are bounded, it readily follows that $\left\{L\left\{y_{k}, \hat{y}_{k}\right\}\right\}$ converges to $L\{y, \hat{y}\}$ in the topology of $S\left(\right.$ i.e. $\left.L^{2}[a, b] \times L^{2}[a, b]\right)$. Hence the sequence $\left\{\left\{y_{k}, \hat{y}_{k}\right\}\right\}$ converges to $\{y, \hat{y}\}$ in the topology of $H^{n}[a, b] \times H^{n}[a, b]$, which implies that the sequence $\left\{\left\{y_{k}, \hat{y}_{k}\right\}\right\}$ converges to $\{y, \hat{y}\}$ in the topology of $H^{n-1}[a, b] \times H^{n-1}[a, b]$ and $\mu\left(\left\{y_{k}, \hat{y}_{k}\right\}-\left\{y, \hat{y}_{k}\right\}\right) \rightarrow 0$ as $k \rightarrow \infty$. Hence $\left\|\{y, \hat{y}\}-\left\{x_{0}, \hat{x}_{0}\right\}\right\|_{H^{n}[a, b] \times H^{n}[a, b]} \leq c$ and

$\mu\left(\{y, \hat{y}\}-\left\{x_{0}, \hat{x}_{0}\right\}\right) \leq r$. Thus $\{y, \hat{y}\} \in V$. Obviously $V$ is a bounded subset of $L^{2}[a, b] \times L^{2}[a, b]$. Thus $V$ is a closed and bounded subset of $L^{2}[a, b] \times L^{2}[a, b]$.

2.3. Operator $T$ and sets $A\left(\left\{x^{*}, \hat{x}^{*}\right\}\right)$ and $A$. For each $\left\{x^{*}, \hat{x}^{*}\right\} \in V$, let $T$ be the operator on $\widetilde{S}_{0}$ defined by

Definition 2.3.

$$
T\{x, \hat{x}\}=\left\{x^{*}, \hat{x}^{*}\right\}+H\left(I-P_{m}\right) N\{x, \hat{x}\}
$$

for $\{x, \hat{x}\} \in \widetilde{S}_{0}$.

We observe that $T$ is well defined on $\widetilde{S}_{0}$.

For each $\left\{x^{*}, \hat{x}^{*}\right\} \in V$, the set $A\left(\left\{x^{*}, \hat{x}^{*}\right\}\right)$ is defined by

Definition 2.4.

$$
A\left(\left\{x^{*}, \hat{x}^{*}\right\}\right)=\left\{\{x, \hat{x}\} \in \widetilde{S}_{0}:\{x, \hat{x}\}=T\{x, \hat{x}\}\right\}
$$


We denote by $A=\cup_{\left\{x^{*}, \hat{x}^{*}\right\} \in V} A\left(\left\{x^{*}, \hat{x}^{*}\right\}\right)$.

Suppose $A\left(\left\{x^{*}, \hat{x}^{*}\right\}\right)$ is non-empty. Then $\{x, \hat{x}\}=T\{x, \hat{x}\}=\left\{x^{*}, \hat{x}^{*}\right\}+H(I-$ $\left.P_{m}\right) N\{x, \hat{x}\}$ for some $X \in \widetilde{S}_{0}$.

Clearly $\{x, \hat{x}\} \in D(L)$ and by Theorem 1.8 we have $Q_{m}\{x, \hat{x}\}=\left\{x^{*}, \hat{x}^{*}\right\}$

Thus

$L\{x, \hat{x}\}=L Q_{m}\{x, \hat{x}\}+L H\left(I-P_{m}\right) N\{x, \hat{x}\}$

using parts of Theorem 1.8 we get

$L\{x, \hat{x}\}-N\{x, \hat{x}\}=P_{m}(L\{x, \hat{x}\}-N\{x, \hat{x}\})$

Hence $\{x, \hat{x}\} \in \widetilde{S}_{0}$ is a solution of the nonlinear BVP if it satisfies th equation

$$
P_{m}(L\{x, \hat{x}\}-N\{x, \hat{x}\})
$$

This equation is called the bifurcation equation of order $m$.

We notice that $L\{x, \hat{x}\}-N\{x, \hat{x}\}=P_{m}(L\{x, \hat{x}\}-N\{x, \hat{x}\})$ on the set $A$ provided $A$ is non-empty. In the following sections we show that $A\left(\left\{x^{*}, \hat{x}^{*}\right\}\right)$ is non-empty. Thus the original MBVP will be reduced to the equivalent bifurcation equation (2.16) on the set $A$.

\subsection{Reduction of the Original MBVP in to an Equivalent Bifurca-} tion Equation using the Schauder's Fixed Point Theorem. The following lemma is needed for our discussions in this section.

Lemma 2.5. Consider the Banach Spaces $H^{n}[a, b] \times H^{n}[a, b]$ and $H^{n-1}[a, b] \times$ $H^{n-1}[a, b]$. Let $\left\{x_{m}, \hat{x}_{m}\right\}$ be a bounded sequence in the space $H^{n}[a, b] \times H^{n}[a, b]$. Then this sequence has a subsequence which converges in the topology of $H^{n-1}[a, b] \times$ $H^{n-1}[a, b]$.

Lemma 2.6. Let all the assumptions stated at the starting of this section and conditions (2.10), (2.11) be satisfied. Then the operator $T: \widetilde{S}_{0} \rightarrow H^{n}[a, b] \times$ $H^{n}[a, b]$ is continuous.

Proof. From the above Lemma we have

$T\{x, \hat{x}\}=\left\{x^{*}, \hat{x}^{*}\right\}+\int_{a}^{b} K_{m}(., s)(N\{x, \hat{x}\}(s)) d s$ for $\{x, \hat{x}\} \in D(N)$.

Suppose $x$ and $y \in \widetilde{S}_{0}$. Then

$T\{x, \hat{x}\}-T\{y, \hat{y}\}=\int_{a}^{b} K_{m}(., s)(N\{x, \hat{x}\}(s)-N\{y, \hat{y}\}(s)) d s$

So

$\sqrt{b-a}\left(\sum_{i=0}^{n-1} \sup _{t \in[a, b]}\left|(T\{x, \hat{x}\}-T\{y, \hat{y}\})^{i}(t)\right|\right)+\left\|(T\{x, \hat{x}\}-T\{y, \hat{y}\})^{n}\right\|$

$=\sqrt{b-a}\left(\sum_{i=0}^{n-1} \sup _{t \in[a, b]}\left|\int_{a}^{b} \frac{\partial^{i} K_{m}(t, s)}{\partial t^{i}}(N\{x, \hat{x}\}(s)-N\{y, \hat{y}\}(s)) d s\right|\right)$

$+\left\|\int_{a}^{b} \frac{\partial^{n} K_{m}(., s)}{\partial t^{n}}(N\{x, \hat{x}\}(s)-N\{y, \hat{y}\}(s)) d s\right\|$

$\leq \sqrt{b-a}\left(\sum_{i=0}^{n-1}\left(\sup _{t \in[a, b]} \int_{a}^{b}\left(\frac{\partial^{i} K_{m}(t, s)}{\partial t^{i}}\right)^{2} d s\right)^{\frac{1}{2}}\right)\|N\{x, \hat{x}\}(s)-N\{y, \hat{y}\}\|+$

$\left(\int_{a}^{b} \int_{a}^{b}\left(\frac{\partial^{n} K_{m}(t, s)}{\partial t^{n}}\right)^{2} d s d t\right)^{\frac{1}{2}}\|N\{x, \hat{x}\}(s)-N\{y, \hat{y}\}\|$

This comes from the Schwartz's inequality

$=\left(\sqrt{b-a}\left(\sum_{i=0}^{n-1} \rho_{m}^{i}\right)+\rho_{m}^{n}\right)\|N\{x, \hat{x}\}(s)-N\{y, \hat{y}\}\|$ 
where

$\rho_{m}^{n}=\left(\int_{a}^{b} \int_{a}^{b}\left(\frac{\partial^{n} K_{m}(t, s)}{\partial t^{n}}\right)^{2} d s d t\right)^{\frac{1}{2}}$

We clearly notice that $\rho_{m}^{n} \rightarrow 0$ as $m \rightarrow \infty$.

Define

$\gamma_{m}=\left(\sqrt{b-a}\left(\sum_{i=0}^{n-1} \rho_{m}^{i}\right)+\rho_{m}^{n}\right)$

Then $\gamma_{m} \rightarrow 0$ as $m \rightarrow \infty$.

Therefore from the above inequality we get that

$$
\begin{aligned}
& \sqrt{b-a}\left(\sum_{i=0}^{n-1} \sup _{t \in[a, b]}\left|(T\{x, \hat{x}\}-T\{y, \hat{y}\})^{i}(t)\right|\right) \\
& +\|T\{x, \hat{x}\}-T\{y, \hat{y}\}\| \\
& \leq \gamma_{m}\|N\{x, \hat{x}\}(s)-N\{y, \hat{y}\}\| \\
& \leq \gamma_{m} k_{0}\|\{x, \hat{x}\}(s)-\{y, \hat{y}\}\|_{H^{n}[a, b] \times H^{n}[a, b]}
\end{aligned}
$$

Hence the map $T$ is continuous.

Corollary 2.7. with all the assumptions in the previous theorem satisfied, the map $T: \widetilde{S}_{0} \rightarrow H^{n-1}[a, b] \times H^{n-1}[a, b]$ is continuous.

Proof. The proof of the corollary follows from the above result and the fact that $\|T\{x, \hat{x}\}\|_{H^{n-1}[a, b] \times H^{n-1}[a, b]} \leq \sqrt{b-a}\left(\sum_{i=0}^{n-1} \sup _{t \in[a, b]}\left|(T\{x, \hat{x}\}-T\{y, \hat{y}\})^{i}(t)\right|\right)+$ $\left\|(T\{x, \hat{x}\}-T\{y, \hat{y}\})^{n}\right\|$

In addition to the previous assumptions, only for the present case we assume that $|\{x, \hat{x}\}| \leq K_{0}$. Thus for $\{x, \hat{x}\} \in \widetilde{S}_{0}$ we have $|N\{x, \hat{x}\}| \leq k_{0}$ and hence

$$
\|N\{x, \hat{x}\}\| \leq k_{0} \sqrt{b-a}
$$

Lemma 2.8. Suppose all the assumptions of this section and (2.10), (2.11),(2.17) are satisfied. Then the set $T\left(\widetilde{S}_{0}\right)$ is relatively compact in $H^{n}[a, b] \times H^{n}[a, b]$.

Proof. Firstly we observe that $T\left(\widetilde{S}_{0}\right)$ is bounded in $H^{n}[a, b] \times H^{n}[a, b]$.

Indeed, let $\{x, \hat{x}\} \in \widetilde{S}_{0}$. Then we have

$T\{x, \hat{x}\}=\left\{x^{*}, \hat{x}^{*}\right\}+\int_{a}^{b} K_{m}(., s)[N\{x, \hat{x}\}] d s$

Therefore

$\sqrt{b-a}\left(\sum_{i=0}^{n-1} \sup _{t \in[a, b]}\left|(T\{x, \hat{x}\})^{i}(t)\right|\right)+\left\|(T\{x, \hat{x}\})^{n}\right\|$

$\leq \sqrt{b-a}\left(\sum_{i=0}^{n-1} \sup _{t \in[a, b]}\left|\left(\left\{x^{*}, \hat{x}^{*}\right\}\right)^{i}(t)\right|\right)+\left\|\left(\left\{x^{*}, \hat{x}^{*}\right\}\right)^{n}\right\|+$

$\sqrt{b-a}\left(\sum_{i=0}^{n-1} \sup _{t \in[a, b]}\left|\int_{a}^{b} \frac{\partial^{i} K_{m}(t, s)}{\partial t^{i}}[N\{x, \hat{x}\}(s)] d s\right|\right)+$

$\left\|\int_{a}^{b} \frac{\partial^{n} K_{m}(t, s)}{\partial t^{n}}[N\{x, \hat{x}\}(s)] d s\right\|$

then, by a simple calculation as in the previous proof, we get

$$
\begin{aligned}
& \sqrt{b-a}\left(\sum_{i=0}^{n-1} \sup _{t \in[a, b]}\left|(T\{x, \hat{x}\})^{i}(t)\right|\right)+\left\|(T\{x, \hat{x}\})^{n}\right\| \\
\leq & \sqrt{b-a}\left(\sum_{i=0}^{n-1} \sup _{t \in[a, b]}\left|\left(\left\{x^{*}, \hat{x}^{*}\right\}\right)^{i}(t)\right|\right)+\left\|\left(\left\{x^{*}, \hat{x}^{*}\right\}\right)^{n}\right\|+\gamma_{m}\|N\{x, \hat{x}\}\| \\
\leq & \sqrt{b-a}\left(\sum_{i=0}^{n-1} \sup _{t \in[a, b]}\left|\left(\left\{x^{*}, \hat{x}^{*}\right\}\right)^{i}(t)\right|\right)+\left\|\left(\left\{x^{*}, \hat{x}^{*}\right\}\right)^{n}\right\|+\gamma_{m} k_{0} \sqrt{b-a}
\end{aligned}
$$

We notice that the right hand side of the above inequality is independent of 
$\{x, \hat{x}\}$. Therefore the set $T\left(\widetilde{S_{0}}\right)$ is bounded in $H^{n}[a, b] \times H^{n}[a, b]$. Hence from the Lemma 2.5 , the set $T\left(\widetilde{S}_{0}\right)$ is relatively compact in $H^{n-1}[a, b] \times H^{n-1}[a, b]$. This completes the proof.

We now present a theorem which reduces the original MBVP to an equivalent bifurcation equation by making use of the Schauder's fixed point theorem.

Theorem 2.9. Let all the assumptions in this section and conditions (2.10), (2.11) and (2.17) be true. Let $m$ be sufficiently large such that

$$
c \leq d-\theta_{m} k_{0} \sqrt{b-a}, r \leq \bar{R}-\bar{\theta}_{m} k_{0} \sqrt{b-a}
$$

Then for each $\left\{x^{*}, \hat{x}^{*}\right\} \in V$ the set $A\left(\left\{x^{*}, \hat{x}^{*}\right\}\right)$ is non-empty. Moreover $L\{x, \hat{x}\}-$ $N\{x, \hat{x}\}=P_{m}(L\{x, \hat{x}\}-N\{x, \hat{x}\})$ on the set $A$.

Proof. By (2.15), it is enough to show that the map $T$ corresponding to $\left\{x^{*}, \hat{x}^{*}\right\} \in$ $V$ has a fixed point in $\widetilde{S}_{0}$. We have seen in the previous theorems that the map $T$ is continuous and the set $T\left(\widetilde{S}_{0}\right)$ is relatively compact in $H^{n-1}[a, b] \times H^{n-1}[a, b]$. We now prove that $T\left(\widetilde{S}_{0}\right) \subset \widetilde{S}_{0}$

Let $\{x, \hat{x}\} \in \widetilde{S}_{0}$, then $T\{x, \hat{x}\} \in H^{n}[a, b] \times H^{n}[a, b]$ and

$T\{x, \hat{x}\}=\left\{x^{*}, \hat{x}^{*}\right\}+\int_{a}^{b} K_{m}(., s)[N\{x, \hat{x}\}(s)] d s$

Therefore

$T\{x, \hat{x}\}-\left\{x_{0}, \hat{x}_{0}\right\}=\left\{x^{*}, \hat{x}^{*}\right\}-\left\{x_{0}, \hat{x}_{0}\right\}+\int_{a}^{b} K_{m}(., s)[N\{x, \hat{x}\}(s)] d s$

Hence

$\left\|T\{x, \hat{x}\}-\left\{x_{0}, \hat{x}_{0}\right\}\right\|_{H^{n}[a, b] \times H^{n}[a, b]}=\left\|\left\{x^{*}, \hat{x}^{*}\right\}-\left\{x_{0}, \hat{x}_{0}\right\}\right\|_{H^{n}[a, b] \times H^{n}[a, b]}$

$+\left\|\int_{a}^{b} K_{m}(., s)[N\{x, \hat{x}\}(s)] d s\right\|_{H^{n}[a, b] \times H^{n}[a, b]}$

$\leq c+\theta_{m}\|N\{x, \hat{x}\}\|$

$\leq c+\theta_{m} k_{0} \sqrt{b-a}$

$\leq d$

Also

$\mu\left(T\{x, \hat{x}\}-\left\{x_{0}, \hat{x}_{0}\right\}\right) \leq \mu\left(\left\{x^{*}, \hat{x}^{*}\right\}-\left\{x_{0}, \hat{x}_{0}\right\}\right)+\mu\left(\int_{a}^{b} K_{m}(., s)[N\{x, \hat{x}\}] d s\right)$

$\leq r+\bar{\theta}_{m}\|N\{x, \hat{x}\}\|$

$\leq r+\bar{\theta}_{m} k_{0} \sqrt{b-a}$

$<\bar{R}$

Thus $T\left(\widetilde{S}_{0}\right) \subset \widetilde{S}_{0}$

We also observed that $\widetilde{S}_{0}$ is a closed, bounded and convex subset of $H^{n-1}[a, b] \times$ $H^{n-1}[a, b]$. Hence the application of Schauder's fixed point theorem to the pair $\widetilde{S}_{0}$ and $T$ yields that the map $T$ has a fixed point in $\widetilde{S}_{0}$. Moreover, since $A\left(\left\{x^{*}, \hat{x}^{*}\right\}\right)$ is non-empty. So, thus we have the desired reduction on the non-empty set $A$.

So, We notice that $L\{x, \hat{x}\}-N\{x, \hat{x}\}=P_{m}(L\{x, \hat{x}\}-N\{x, \hat{x}\})$ on the set $A$ since $A$ is non-empty.

\section{ACKNOWLEDGEMENTS}

This study is funded under the Research Project SR/S4/MS:279/05, by DST, Ministry of Science and Technology, Govt. of INDIA. 


\section{REFERENCES}

[1] A. Aliouche, Common Fixed Point Theorems for Hybrid Mappings Satysfying Generalised Contractive Conditions, Journal of Nonlinear Sciences and Applications, 2 (2009), 136-145.

[2] A. Azam, M. Arshad and I. Beg, Common Fixed Point Theorems in cone Metric Spaces, Journal of Nonlinear Sciences and Applications, 1 (2009), 204-213.

[3] L. Cesari, Functional analysis and Galerkin's Method, Mich. Math J., 11 (1964), 385-359.

[4] L. Cesari and R. Kannan, Functional analysis and nonlinear differential equations, Contributions to differential equations, vol 1, No 2, Interscience, Newyork, 1956.

[5] P.C. Das and M. Venkatesulu, An Alternative Method for Boundary Value Problems with Ordinary Differential Equations, Riv. Math. Univ. Parma, 4 (1983), 15-25.

[6] P.C. Das and M. Venkatesulu, An Existential Analysis for a Nonlinear Differential Equation with Nonlinear Multipoint Boundary Conditions via the alternative mathod, Att. Sem. Mat. Fis.Univ, Modena, XXXII (1983), 287-307.

[7] P.C. Das and M. Venkatesulu, An Existential Analysis for a Multipoint Boundary Value Problem via Alternative Method, Riv. Math. Univ. Parma, 4 (1984), 183-193.

[8] L. Kantarovich, Functional Analysis and Applied Mathematics, Uspehi Mat.Nauk, 3 (1948), 89-185.

[9] J. Locker, Functional Analysis and Two Point Differential operators, Longman scientific and Technical, Harlow, 1986.

[10] J. Locker, An existential analysis for nonlinear boundary value problems, SIAM J. Appl. Math., 19 (1970), 109-207.

[11] P. K. Baruah, B.V.K. Bharadwaj and M. Venkatesulu, Characterization of Maximal, Minimal and Inverse Operators and an Existence Theorem for Coupled Ordinary Differential Operator, International Journal of Mathematics and Analysis, 1, January-June, (2009), 25-56.

[12] P. K. Baruah, B.V.K. Bharadwaj and M. Venkatesulu, Green's Matrix for Linear Coupled Ordinary Differential Operator, International Journal of Mathematics and Analysis, 1(1), January-June, (2009), pp 57-82.

[13] R. Ali Khan and N. Ahmad Asif, Positive Solutions for a Class of Singular Two Point Boundary Value Problems, Journal of Nonlinear Sciences and Applications, 2 (2009), 126135.

[14] S. Sinha, O. P. Mishra and J. Dhar, Study of a prey-predator dynamics under the simultaneous effectof toxicant and disease, The Journal of Nonlinear Sciences and Applications, 1 (2008), 36-44.

[15] M. Venkatesulu, P. K. Baruah and A. Prabu, A Existence theorem for IVPs of Coupled Ordinary Differential Equations, International Journal of Differential Equations and Applications, 10 (2005), 435-447.

1,2 Department of Mathematics and Computer Science, Sri Sathya Sai UniverSity, Prashanthi Nilayam, Puttaparthy, INDIA.

E-mail address: baruahpk@sssu.edu.in, bvkbharadwaj@sssu.edu.in

3 Department of Mathematics and Computer Applications, Kalasalingam UniVERsity, Krishnankoil, TAMILnAdU, INDIA

E-mail address: venkatesulum_2000@yahoo.co.in 